\title{
Dispersion in a Pulsatile Electroosmotic Flow Affected by Wall Slippage
}

\author{
J. E. Muñoz ${ }^{1}$, J. C. Arcos ${ }^{1}$, O. E. Bautista ${ }^{1}$, E. G. Bautista ${ }^{1}$, F. Méndez ${ }^{2}$ \\ ${ }^{1}$ ESIME Azcapotzalco, Instituto Politécnico Nacional, \\ Av. de las Granjas No. 682, Col. Santa Catarina, Azcapotzalco, Ciudad de México 02250, Mexico \\ jcahdez@hotmail.com; obautista@ipn.mx; erbautista@ipn.mx \\ ${ }^{2}$ Facultad de Ingeniería, UNAM. Coyoacan, Ciudad de México 04510, Mexico \\ fmendez@unam.mx
}

\section{Extended Abstract}

The dispersion of a non-reacting solute released in a pulsatile electroosmotic flow (PEOF) in a microcapillary is analytically studied. In this work, we take into account the slippage condition on the wall of the microcapillary. The results obtained for the dispersion coefficient are function of the dimensionless slip length $\delta$, an angular Reynolds number $R_{\omega}$, the amplitude $\xi$ of the oscillatory component of the external electric field and an electrokinetic parameter $\kappa$ that relates the radius of the microcapillary with the Debye length. In this study we show that the presence of the Navier slip condition in a pulsatile electroosmotic flow tends to increase the dispersivity $\mathcal{D}$ of the solute in comparison when the no-slip condition is not considered.

Once upon a time, Aris [1] studied the dispersion of a pressure-driven and periodic flow through a circular tube. The design and construction of Labs-on-a-chip (LOCs), are directly related to the study of electrokinetic phenomena. Those LOCs are of great interest due to the ability to handle low fluid volumes, lower manufacturing costs and flexibility for automated operation. That is why many works have been developed in the field of the AC Electroosmosis for manipulating microflows based on the interaction of the electric double layer (EDL) with an externally applied alternating electric field [2-3]. In this extended abstract, recognizing the inherent potential of PEOFs as an important means for electroosmotic mass flow control/enhancement [4-5] and considering that the surface of microchannel walls can be hydrophobic, we determine the effective dispersion coefficient, also known as Taylor dispersion, in the long time scale of the process, i.e., when the transient stage has died out and the flow field corresponds to the periodic transient motion. Quantitative surprises emerge from this study, showing that the dispersion coefficient can be notably increased due to a PEOF when slippage is considered. The slip condition enhances $\mathcal{D}$ in a PEOF, particularly for $\kappa \gg 1$ and for values of $R_{\omega} \sim \mathcal{O}(10)$ and $\xi=1$. Considering slippage, for the same value of the relative amplitude of the oscillatory electric field, the effective dispersion coefficient can be greater by two orders of magnitude than that where the no-slip condition is assumed. We determined that the time required to provide an uniform concentration of an analyte that was previously injected as a solute band was 4 hours by considering the solute influence of an external pulsatile body force inducing an EOF. However, considering a hydrophobic surface at the boundary wall of the microcapillary allows developing a time of approximately 3 minutes, confirming that the presence of a slip condition cannot be neglected in the PEOF microscale context.

\section{References}

[1] R. Aris, "On the dispersion of a solute in pulsating flowing through a tube," Proc. R. Soc. London Ser. A-Math. Phys. Eng. Sci., vol. 259, pp. 370-376, 1960.

[2] P. Dutta and A. Beskok, "Analytical solution of time periodic electroosmotic flows: analogies to stokes' second problem," Anal. Chem., vol. 73, pp. 5097-5102, 2001.

[3] N. G. Green, A. Ramos, A. Gonzalez, H. Morgan and A. Castellanos, "Fluid flow induced by nonuniform ac electric fields in electrolytes on microelectrodes. I. Experimental measurements," Phys. Rev., vol. 61, pp. 4011-4018, 2000.

[4] G. Rojas and J. Arcos and M. Peralta and F. Méndez and O. Bautista, "Pulsatile electroosmotic flow in a microcapillary with the slip boundary condition," Colloid Surf. A-Physicochem. Eng. Asp, vol. 513, pp. 57-65, 2017.

[5] S. Chakraborty and S. Ray, "Mass flow-rate control through time periodic electro-osmotic flows in circular microchannels," Phys. Fluids, vol. 20, no. 083602, pp. 1-11, 2008. 